hep-th/0401170

SOGANG-HEP 306/03

\title{
Symplectic Embedding of a Massive Vector-Tensor Theory with Topological Coupling
}

\author{
Yong-Wan Kim ${ }^{1}$ and Chang-Yeong Lee ${ }^{2}$ \\ Department of Physics and Institute for Science and Technology, \\ Sejong University, Seoul 143-747, Korea \\ Seung-Kook Kim ${ }^{3}$ \\ Department of Physics, Seonam University, Chonbuk 590-170, Korea \\ Young-Jai Park ${ }^{4}$ \\ Department of Physics, Sogang University, Seoul 121-742, Korea
}

(October 30, 2018)

\begin{abstract}
In the symplectic Lagrangian framework we newly embed an irreducible massive vector-tensor theory into a gauge invariant system, which has become reducible, by extending the configuration space to include an additional pair of scalar and vector fields, which give the desired Wess-Zumino action. A comparision with the BFT Hamiltonian embedding approach is also done.
\end{abstract}

PACS: 11.10.Ef, 11.30.Ly, 11.15.-q

Keywords: Symplectic embedding; Antisymmetric tensor field;

Reducible; Topological coupling

\footnotetext{
${ }^{1}$ Electronic address: ywkim@sejong.ac.kr

${ }^{2}$ Electronic address: cylee@sejong.ac.kr

${ }^{3}$ Electronic address: skandjh@empal.com

${ }^{4}$ Electronic address: yjpark@sogang.ac.kr
} 


\section{Introduction}

The Dirac quantization method [1] has been widely used in order to quantize Hamiltonian system involving first- and second-class constraints. However, the resulting Dirac brackets may be field-dependent and nonlocal, and thus pose serious ordering problems. The BRST quantization [2] on the lines of Dirac established by Batalin, Fradkin, and Vilkovisky [3], and then improved in a more tractable and elegant Hamiltonian embedding by Batalin, Fradkin, and Tytin (BFT) [4], does not suffer from these difficulties, as it relies on a simple Poisson bracket structure. As a result, this embedding of second-class system into first-class one has received much attention in the past decade $[5,6,7]$.

While the various quantization methods based on the Hamiltonian formulation are developed for general types of gauge theories, Faddeev and Jackiw [8] have introduced an equivalent scheme based on a first order Lagrangian which does not need to classify constraints as primary, secondary, etc. Since this scheme deals with less number of constraints than that of Dirac, it is proved to be relatively simple to find the Dirac brackets. After this work, there are numerous related analyses $[9,10,11]$. In particular, we had shown that in this framework, a gauge non-invariant theory can be embedded into a gauge invariant one by investigating the properties of the symplectic twoform matrix and its corresponding zero modes [12]. Recently, there are some renewed interests $[13,14,15]$ in the subjects of symplectic embedding including noncommutative theories.

On the other hand, antisymmetric tensor fields, which first appear as a mediator of the string interaction [16], have been much interested in an alternative of the Higgs mechanism $[17,18]$. With the topologically interacting term of the form $B \wedge F$, the physical degree of freedom of the antisymmetric rank two tensor field $B$ is absorbed by the vector field, making it massive. This mechanism is considered generic in string phenomenology [19]. Moreover, various dual descriptions between different theories have been widely studied where the antisymmetric tensor field plays an important role in realization of dualities [20, 21]. These dual relations were also independently confirmed by defining dual operations on the space of pairs of different gauge forms [22]. These were a surprising result that, as like the well-known dual equivalence [23] of the first order self-dual theory and Maxwell-Chern-Simons theory in $d=3$, they have all shown that the first order master action even 
in $d=4$ has a dual relation with the Maxwell-Kalb-Ramond (MKR) theory. Recently, it has shown that through the BFT embedding technique the gauge non-invariant master action is equivalent to the gauge invariant MKR theory [24]. Very recently, we have generalized their results to include both the gauge symmetry breaking term and the topological coupling one [7], resulting in a new type of Wess-Zumino (WZ) action [25] as well as the usual Stückelberg one.

This paper is organized as follows. In section 2, we briefly show the dual relation of the first order master Lagrangian between the Abelian Proca and the Kalb-Ramond (KR) massive theories classically by using the equations of motion. Then, we explicitly carry out the symplectic quantization for this gauge noninvariant Lagrangian. In section 3, we embed this master Lagrangian into a desired gauge invariant one, and make clear the relation between the "trivial" zero modes and the symmetry transformations. We also explicitly show that one of the "trivial" zero modes is related to a reducible constraint. In section 4, we compare the result of the symplectic embedding with the BFT one. Our conclusion is given in section 5 .

\section{Symplectic Quantization of the Master La- grangian}

In this section, we reconsider the first order Lagrangian [21] for the Abelian massive vector and tensor fields, and quantize it explicitly through the symplectic scheme. This Lagrangian is not only known to have the dual relation with the MKR Lagrangian [7, 21, 22, 24], but also plays a role of a master Lagrangian of the Proca and the KR models.

First, let us briefly review that the following first order Lagrangian

$$
\mathcal{L}=-\frac{1}{4} B_{\mu \nu} B^{\mu \nu}+\frac{1}{2} A_{\mu} A^{\mu}+\frac{1}{2 m} \epsilon_{\mu \nu \rho \sigma} B^{\mu \nu} \partial^{\rho} A^{\sigma}
$$

describes classically the massive Proca and KR theories simultaneously, i.e., the Lagrangian (2.1) is a master Lagrangian ${ }^{5}$ of the mentioned two theories. By varying the Lagrangian with the fields $A^{\mu}$ and $B^{\mu \nu}$, one obtains their

\footnotetext{
${ }^{5}$ For a quantum mechanical treatment, see the Ref.[21].
} 
equations of motion as

$$
\begin{aligned}
B_{\mu \nu}-\frac{1}{m} \epsilon_{\mu \nu \rho \sigma} \partial^{\rho} A^{\sigma} & =0 \\
A_{\mu}+\frac{1}{2 m} \epsilon_{\mu \nu \rho \sigma} \partial^{\nu} B^{\rho \sigma} & =0
\end{aligned}
$$

respectively. By eliminating the antisymmetric $B^{\mu \nu}$ fields from the Lagrangian (2.1), the Proca model is obtained as follows

$$
\mathcal{L}_{\text {Proca }}=-\frac{1}{4 m^{2}} F_{\mu \nu} F^{\mu \nu}+\frac{1}{2} A_{\mu} A^{\mu} .
$$

Similarly, by using the equation of motion for the field $A^{\mu}$, one could obtain the KR Lagrangian as

$$
\mathcal{L}_{K R}=\frac{1}{12 m^{2}} G_{\mu \nu \sigma} G^{\mu \nu \sigma}-\frac{1}{4} B_{\mu \nu} B^{\mu \nu} .
$$

This shows that the first order Lagrangian (2.1) is the master Lagrangian of the two massive theories at the classical level.

On the other hand, according to the symplectic scheme [8, 9], it is easy to find their symplectic brackets in a few steps equivalent to the Dirac ones in the Hamiltonian formulation as

$$
\begin{aligned}
\left\{A^{0}(x), A^{i}(y)\right\} & =\partial_{x}^{i} \delta(x-y), \\
\left\{A^{i}(x), \pi_{j}(y)\right\} & =m^{2} \delta_{j}^{i} \delta(x-y)
\end{aligned}
$$

for the Proca model [14] as well as

$$
\begin{aligned}
\left\{B^{0 i}(x), B^{j k}(y)\right\} & =\left(\delta^{i j} \partial_{x}^{k}-\delta^{i k} \partial_{x}^{j}\right) \delta(x-y), \\
\left\{B^{i j}(x), \pi_{k l}(y)\right\} & =m^{2}\left(\delta_{k}^{i} \delta_{l}^{j}-\delta_{k}^{j} \delta_{l}^{i}\right) \delta(x-y)
\end{aligned}
$$

for the KR theory [26].

Now, in order to implement the usual symplectic quantization of the master Lagrangian itself which is composed of two kinds of the fields with different ranks as well as their topological coupling term, we rewrite the Lagrangian to an alternating first-ordered one from symmetrized form of Eq. (2.1) as

$$
\mathcal{L}^{(0)}=\frac{1}{4 m} \epsilon_{i j k} B^{j k} \dot{A}^{i}-\frac{1}{4 m} \epsilon_{i j k} A^{k} \dot{B}^{i j}-\mathcal{H}^{(0)}
$$


where the Hamiltonian is

$$
\mathcal{H}^{(0)}=\frac{1}{4} B_{\mu \nu} B^{\mu \nu}-\frac{1}{2} A_{\mu} A^{\mu}-\frac{1}{m} \epsilon_{i j k} B^{0 i} \partial^{j} A^{k}-\frac{1}{2 m} A^{0} \epsilon_{i j k} \partial^{i} B^{j k} .
$$

Here, we adopt the conventions: $\epsilon_{0 i j k}=\epsilon_{i j k}, \epsilon^{123}=+1$, and $g^{\mu \nu}=(+---)$. As is clear in the Lagrangian (2.7), there are no needs to introduce additional auxiliary fields such as momenta because it already has the form of first order.

Then, we identify the initial sets of symplectic variables and their conjugate momenta from the first order Lagrangian as follows

$$
\begin{aligned}
\xi^{(0) \alpha} & =\left(A^{i}, B^{i j}, A^{0}, B^{0 i}\right), \\
a_{\alpha}^{(0)} & =\left(\frac{1}{4 m} \epsilon_{i j k} B^{j k},-\frac{1}{2 m} \epsilon_{i j k} A^{k}, 0, \overrightarrow{0}^{T}\right) .
\end{aligned}
$$

Note that the coefficients of the fields having time derivative in the canonical sector play the roles of momenta, and $B^{i j}$ in the symplectic variable $\xi^{(0) \alpha}$ denote the independent component fields such as $B^{i j}:=\left(B^{12}, B^{23}, B^{31}\right)$ in order, collectively, while any contracted indices are understood to be summed over unless otherwise mentioned. From the definition of the symplectic twoform matrix [9]:

$$
f_{\alpha \beta}(x, y)=\frac{\partial a_{\beta}(y)}{\partial \xi^{\alpha}(x)}-\frac{\partial a_{\alpha}(x)}{\partial \xi^{\beta}(y)},
$$

we explicitly obtain the following zeroth-iterated matrix

$$
f_{\alpha \beta}^{(0)}(x, y)=\left(\begin{array}{cccc}
O & \frac{1}{m} \epsilon_{i(j k)} & \overrightarrow{0} & O \\
-\frac{1}{m^{2} \epsilon_{(i j) k}} & O & \overrightarrow{0} & O \\
\overrightarrow{0}^{T} & \overrightarrow{0}^{T} & 0 & \overrightarrow{0}^{T} \\
O & O & \overrightarrow{0} & O
\end{array}\right) \delta(x-y) .
$$

The tensor components $\epsilon_{i(j k)}, \epsilon_{(i j) k}$ are given by

$$
\epsilon_{i(j k)}=\left(\begin{array}{ccc}
0 & 1 & 0 \\
0 & 0 & 1 \\
1 & 0 & 0
\end{array}\right),
$$

where the $i / j k$-components outside/inside the parenthesis denote explicitly the vector/tensor fields, and behave as like the totally antisymmetric tensor $\epsilon_{i j k}$, similar for $\epsilon_{(i j) k}$. Thereinafter, let us omit the parenthesis otherwise a 
confusion arises. We also denote that in the matrix $f_{\alpha \beta}^{(0)}$ the $O, \overrightarrow{0}$ and $\overrightarrow{0}^{T}$ symbols stand for a $3 \times 3$ null matrix, a three-dimensional column null vector, and its transpose, respectively. Since we easily know that the matrix $f_{\alpha \beta}^{(0)}$ is singular, there exist four-fold infinities related to zero modes, $\tilde{\nu}_{\alpha}^{(0) T}(\sigma, x)$, labelled by discrete indices $\sigma=\left(\epsilon_{1}, \vec{\epsilon}_{2}\right)$ with $\vec{\epsilon}_{2} \equiv\left(\left(\epsilon_{2}^{1}, 0,0\right),\left(0, \epsilon_{2}^{2}, 0\right),\left(0,0, \epsilon_{2}^{3}\right)\right)$, where $\epsilon_{1}$ and $\vec{\epsilon}_{2}$ are arbitrary functions of the continuum label $x$, explicitly with components

$$
\begin{aligned}
& \tilde{\nu}_{\alpha}^{(0) T}\left(\epsilon_{1}, x\right)=\left(\overrightarrow{0}, \overrightarrow{0}, \epsilon_{1}, \overrightarrow{0}\right), \\
& \tilde{\nu}_{\alpha}^{(0) T}\left(\vec{\epsilon}_{2}, x\right)=\left(\overrightarrow{0}, \overrightarrow{0}, 0, \vec{\epsilon}_{2}\right) .
\end{aligned}
$$

Therefore, the four-fold zero modes $\tilde{\nu}_{\alpha}^{(0) T}(\sigma, x)$ generate four corresponding Lagrangian constraints $\Omega_{\epsilon_{1}}$ and $\Omega_{\vec{\epsilon}_{2}}$ defined as

$$
\int d x \Omega_{\sigma}(x)=\int d x \tilde{\nu}_{\alpha}^{(0) T}(\sigma, x) \frac{\partial}{\partial \xi^{(0) \alpha}(x)} \int d y \mathcal{H}^{(0)}(y)=0,
$$

such that

$$
\begin{aligned}
& \Omega_{\epsilon_{1}}=A_{0}+\frac{1}{2 m} \epsilon_{i j k} \partial^{i} B^{j k}=0, \\
& \Omega_{\vec{\epsilon}_{2}}=B_{0 i}-\frac{1}{m} \epsilon_{i j k} \partial^{j} A^{k}=0 .
\end{aligned}
$$

These constraints should be conserved in time, which requirement is incorporated into the Lagrangian (2.7), resulting in an extension of the symplectic space with auxiliary fields, $\alpha, \beta^{i}$, which correspond to Lagrange multipliers.

As a result, the first iterated Lagrangian is written as

$$
\mathcal{L}^{(1)}=\frac{1}{4 m} \epsilon_{i j k} \dot{A}^{i} B^{j k}-\frac{1}{4 m} \epsilon_{i j k} \dot{B}^{i j} A^{k}+\Omega_{\epsilon_{1}} \dot{\alpha}+\Omega_{\epsilon_{2}^{i}} \dot{\beta}^{i}-\mathcal{H}^{(1)},
$$

where the first-iterated Hamiltonian is given by

$$
\begin{aligned}
\mathcal{H}^{(1)}(\xi) & =\left.\mathcal{H}^{(0)}\right|_{\Omega_{\sigma}=0} \\
& =\frac{1}{4} B_{i j} B^{i j}-\frac{1}{2} A_{i} A^{i}-\frac{1}{2} B_{0 i} B^{0 i}+\frac{1}{2} A_{0} A^{0} .
\end{aligned}
$$

We have now for new symplectic variables and their conjugate momenta as

$$
\begin{aligned}
\xi^{(1) \alpha} & =\left\{A^{i}, B^{i j}, A^{0}, B^{0 i}, \alpha, \beta^{i}\right\} \\
a_{\alpha}^{(1)} & =\left\{\frac{1}{4 m} \epsilon_{i j k} B^{j k},-\frac{1}{2 m} \epsilon_{i j k} A^{k}, 0, \overrightarrow{0}^{T}, \Omega_{\epsilon_{1}}, \Omega_{\epsilon_{2}^{i}}\right\} .
\end{aligned}
$$


The first-iterated symplectic matrix is again obtained as

$$
f_{\alpha \beta}^{(1)}(x, y)=\left(\begin{array}{cccccc}
O & \frac{1}{m} \epsilon_{i j k} & \overrightarrow{0} & O & \overrightarrow{0} & \frac{1}{m} \epsilon_{i j k} \partial^{k} \\
-\frac{1}{m} \epsilon_{i j k} & O & \overrightarrow{0} & O & -\frac{1}{m} \epsilon_{i j k} \partial^{k} & O \\
\overrightarrow{0}^{T} & \overrightarrow{0} & 0 & \overrightarrow{0}^{T} & 1 & \overrightarrow{0}^{T} \\
O & O & \overrightarrow{0} & O & \overrightarrow{0} & -\delta_{i j} \\
\overrightarrow{0}^{T} & -\frac{1}{m} \epsilon_{i j k} \partial^{k} & -1 & \overrightarrow{0}^{T} & 0 & \overrightarrow{0}^{T} \\
\frac{1}{m} \epsilon_{i j k} \partial^{k} & O & \overrightarrow{0} & \delta_{i j} & \overrightarrow{0} & O
\end{array}\right) \delta(x-y) .
$$

One can now easily see that this matrix has an inverse as

$$
\left(f_{\alpha \beta}^{(1)}\right)^{-1}(x, y)=\left(\begin{array}{cccccc}
O & -m \epsilon_{i j k} & \partial^{i} & O & \overrightarrow{0} & O \\
m \epsilon_{i j k} & O & \overrightarrow{0} & F_{24} & \overrightarrow{0} & O \\
\partial^{i} & \overrightarrow{0} & 0 & \overrightarrow{0}^{T} & -1 & \overrightarrow{0}^{T} \\
O & F_{24}^{T} & \overrightarrow{0} & O & \overrightarrow{0} & \delta_{i j} \\
\overrightarrow{0}^{T} & \overrightarrow{0}^{T} & 1 & \overrightarrow{0}^{T} & 0 & \overrightarrow{0}^{T} \\
O & O & \overrightarrow{0} & -\delta_{i j} & \overrightarrow{0} & O
\end{array}\right) \delta(x-y),
$$

where

$$
F_{24}=\left(\begin{array}{ccc}
\partial^{2} & -\partial^{1} & 0 \\
0 & \partial^{3} & -\partial^{2} \\
-\partial^{3} & 0 & \partial^{1}
\end{array}\right) .
$$

Since there are no more new non-trivial zero modes, the iteration process is stopped at this stage. From this inverse matrix we easily read the desired nonvanishing symplectic brackets for the vector and the tensor fields as

$$
\begin{aligned}
\left\{A^{0}(x), A^{i}(y)\right\} & =\partial_{x}^{i} \delta(x-y), \\
\left\{A^{i}(x), B^{j k}(y)\right\} & =m \epsilon^{i j k} \delta(x-y), \\
\left\{B^{0 i}(x), B^{j k}(y)\right\} & =\left(\delta^{i j} \partial_{x}^{i}-\delta^{i k} \partial_{x}^{j}\right) \delta(x-y) .
\end{aligned}
$$

This ends the usual symplectic procedure with the only four true constraints in Eq. (2.14).

Comparing this symplectic scheme with the Dirac formulation, one easily sees that the former has less number of the constraints than that of the latter which has 14 constraints shown in Eqs. (4.2) and (4.3) in section 4, does not need to define primary constraints, and thus more efficient to get the brackets. In this respect, it is generally understood that the symplectic method deals only with true constraints while Dirac's one over-constrained. 


\section{Gauge Invariant Symplectic Embedding}

In this section, we embed the first order master Lagrangian without resorting to the Hamiltonian method, which is the usual way of getting corresponding gauge invariant Lagrangian. In the usual BFT embedding scheme, one first works with full Dirac constraints and Hamiltonian defined in the phase space, converts them into gauge invariant ones through the systematic BFT scheme in an extended phase space, and finally using the path integral methods performs a series of integrations for momentum variables to find out a corresponding gauge invariant Lagrangian in an extended configuration space. Compared with this rather long procedure, the gauge invariant symplectic embedding scheme has its merit of simplicity based on the singular property of the symplectic matrix with corresponding "trivial" zero modes which will be defined shortly.

The idea is simply to consider that a desired gauge invariant Lagrangian resulting from an embedding procedure would be provided by

$$
\mathcal{L}_{T}=\mathcal{L}_{O}+\mathcal{L}_{W Z}
$$

where the Lagrangian $\mathcal{L}_{O}$ is the symmetrized original master Lagrangian $\mathcal{L}^{(0)}$ in Eq. (2.7). Then, the symplectic procedure is greatly simplified, if we make the following "educated" guess for WZ Lagrangian ${ }^{6}$, respecting the Lorentz symmetry,

$$
\mathcal{L}_{W Z}=c_{1} \partial_{\mu} \theta \partial^{\mu} \theta+c_{2} A^{\mu} \partial_{\mu} \theta+c_{3} Q_{\mu \nu} Q^{\mu \nu}+c_{4} B_{\mu \nu} Q^{\mu \nu}
$$

where $Q_{\mu \nu}=\partial_{\mu} Q_{\nu}-\partial_{\nu} Q_{\mu}$. Here $\theta$ and $Q^{\mu}$ are gauge degrees of freedom with respect to the original fields $A^{\mu}$ and $B^{\mu \nu}$, respectively. As an Ansatz for a consistent Lorentz covariant embedding, we shall take the coefficients $c_{1}, c_{2}, c_{3}, c_{4}$ to be constants, and we will fix these by the condition that a finally iterated symplectic matrix has "trivial" zero modes on the one hand

\footnotetext{
${ }^{6}$ Similar to the Proca model [14], one could consider the Ansatz more general and manifestly Lorentz invariant functions of WZ Lagrangian composed of a scalar $\theta$ and vector fields $Q^{\mu}$, however those consideration only adds unnecessary calculational complication. On the other hand, in recent works [15], there makes an attempt on the Lagrangian embedding a bit systematic, however in practice it seems very complicated even in the simplest one-form cases $[12,13,14]$ including the Proca model. Furthermore, it may be intractable to apply the method to reducible systems, including the massive vector-tensor theory with topological coupling.
} 
and these zero modes should generate no new constraints on the other hand. Furthermore, we will show that the "trivial" zero modes related with the finally iterated symplectic matrix correctly yield proper rules of gauge transformation including a first-stage reducible trivial zero mode associated with a reducible constraint.

Now, we could read the canonical momenta of the total Lagrangian as

$$
\begin{array}{ll}
\pi_{0}=-c_{2} \theta, & \pi_{\theta}=2 c_{1} \dot{\theta} \\
\pi_{0 i}=-2 c_{4} Q_{i}, & P_{i}=4 c_{3}\left(\dot{Q}_{i}-\partial_{i} Q_{0}\right) .
\end{array}
$$

Here, we have used the partially integrated Lagrangian for the second and fourth terms in Eq. (3.2) in order to easily show the coincidence with the constraints obtained from the BFT embedding in section 4. Along with these auxiliary variables (momenta), to implement the symplectic procedure as like in the previous section, we write the total Lagrangian (3.1) in the first-ordered form as

$$
\mathcal{L}_{T}^{(0)}=\frac{1}{4 m} \epsilon_{i j k} B^{j k} \dot{A}^{i}-\frac{1}{4 m} \epsilon_{i j k} A^{k} \dot{B}^{i j}+\pi_{0} \dot{A}^{0}+\pi_{\theta} \dot{\theta}+\pi_{0 i} \dot{B}^{0 i}+P_{i} \dot{Q}^{i}-\mathcal{H}_{T}^{(0)},
$$

where the Hamiltonian is given by

$$
\begin{aligned}
\mathcal{H}_{T}^{(0)} & =\frac{1}{4} B_{\mu \nu} B^{\mu \nu}-\frac{1}{2} A_{\mu} A^{\mu}-\frac{1}{m} \epsilon_{i j k} B^{0 i} \partial^{j} A^{k}-\frac{1}{2 m} A^{0} \epsilon_{i j k} \partial^{i} B^{j k} \\
& +\frac{1}{4 c_{1}} \pi_{\theta}^{2}+\frac{1}{8 c_{3}} P_{i} P^{i}-c_{1} \partial_{i} \theta \partial^{i} \theta-c_{2} A^{i} \partial_{i} \theta-c_{3} Q_{i j} Q^{i j}-c_{4} B_{i j} Q^{i j} \\
& +P_{i} \partial^{i} Q^{0}+2 c_{4} B^{0 i} \partial_{i} Q_{0} .
\end{aligned}
$$

Then, the initial set of symplectic variables $\xi^{(0) \alpha}$ and their conjugate momenta $a_{\alpha}^{(0)}$ are given by

$$
\begin{aligned}
\xi^{(0) \alpha} & =\left(A^{i}, B^{i j}, \theta, \pi_{\theta}, Q^{i}, P_{i}, A^{0}, B^{0 i}, Q^{0}\right) \\
a_{\alpha}^{(0)} & =\left(\frac{1}{4 m} \epsilon_{i j k} B^{j k},-\frac{1}{2 m} \epsilon_{i j k} A^{k}, \pi_{\theta}, 0, P_{i}, \overrightarrow{0}^{T},-c_{2} \theta,-2 c_{4} Q_{i}, 0\right) .
\end{aligned}
$$

From the above set of the symplectic variables we read off the symplectic 
matrix defined in Eq. (2.10) to be

$$
f_{\alpha \beta}^{(0)}(x, y)=\left(\begin{array}{ccccccccc}
O & \frac{1}{m} \epsilon_{i j k} & \overrightarrow{0} & \overrightarrow{0} & O & O & \overrightarrow{0} & O & \overrightarrow{0} \\
-\frac{1}{m} \epsilon_{i j k} & O & \overrightarrow{0} & \overrightarrow{0} & O & O & \overrightarrow{0} & O & \overrightarrow{0} \\
\overrightarrow{0}^{T} & \overrightarrow{0^{T}} & 0 & -1 & \overrightarrow{0}^{T} & \overrightarrow{0}^{T} & -c_{2} & \overrightarrow{0}^{T} & 0 \\
\overrightarrow{0}^{T} & \overrightarrow{0}^{T} & 1 & 0 & \overrightarrow{0}^{T} & \overrightarrow{0}^{T} & 0 & \overrightarrow{0}^{T} & 0 \\
O & O & \overrightarrow{0} & \overrightarrow{0} & O & -\delta_{i j} & \overrightarrow{0} & 2 c_{4} \delta_{i j} & \overrightarrow{0} \\
O & O & \overrightarrow{0} & \overrightarrow{0} & \delta_{i j} & O & \overrightarrow{0} & O & \overrightarrow{0} \\
\overrightarrow{0}^{T} & \overrightarrow{0}^{T} & c_{2} & 0 & \overrightarrow{0}^{T} & \overrightarrow{0}^{T} & 0 & \overrightarrow{0}^{T} & 0 \\
O & O & \overrightarrow{0} & \overrightarrow{0} & -2 c_{4} \delta_{i j} & O & \overrightarrow{0} & O & 0 \\
O & O & \overrightarrow{0} & \overrightarrow{0} & O & O & \overrightarrow{0} & O & \overrightarrow{0}
\end{array}\right) \delta(x-y),
$$

which is manifestly singular as we observe the last null row and column, and thus the matrix has non-trivial zero modes given by

$$
\begin{aligned}
& \nu_{\alpha}^{(0) T}\left(\epsilon_{1}, x\right)=\left(\overrightarrow{0}^{T}, \overrightarrow{0}^{T}, 0,-c_{2} \epsilon_{1}, \overrightarrow{0}^{T}, \overrightarrow{0}^{T}, \epsilon_{1}, \overrightarrow{0}^{T}, 0\right), \\
& \nu_{\alpha}^{(0) T}\left(\vec{\epsilon}_{2}, x\right)=\left(\overrightarrow{0}^{T}, \overrightarrow{0}^{T}, 0,0, \overrightarrow{0}^{T}, 2 c_{4} \vec{\epsilon}_{2}, 0, \overrightarrow{\epsilon_{2}}, 0\right), \\
& \nu_{\alpha}^{(0) T}\left(\epsilon_{3}, x\right)=\left(\overrightarrow{0}^{T}, \overrightarrow{0}^{T}, 0,0, \overrightarrow{0}^{T}, \overrightarrow{0}^{T}, 0, \overrightarrow{0}^{T}, \epsilon_{3}\right) .
\end{aligned}
$$

Applying these zero-modes from the left to the equation of motion we have obtained constraints $\phi_{\sigma} \equiv\left(\phi_{\epsilon_{1}}, \phi_{\vec{\epsilon}_{2}}, \phi_{\epsilon_{3}}\right)$ as

$$
\begin{aligned}
& \phi_{\epsilon_{1}}=A_{0}+\frac{1}{2 m} \epsilon_{i j k} \partial^{i} B^{j k}+\frac{c_{2}}{2 c_{1}} \pi_{\theta}=0, \\
& \phi_{\vec{\epsilon}_{2}}=B_{0 i}-\frac{1}{m} \epsilon_{i j k} \partial^{j} A^{k}-\frac{c_{4}}{2 c_{3}} P_{i}=0, \\
& \phi_{\epsilon_{3}}=\partial^{i} P_{i}+2 c_{4} \partial_{i} B^{0 i}=0 .
\end{aligned}
$$

Next, following the symplectic algorithm for theories having gauge symmetry $[9,12,14]$, we obtain the first-iterated Lagrangian by enlarging the canonical sector with the constraints $\phi_{\sigma}$ and their associated Lagrangian multipliers $\left(\alpha, \beta^{i}, \gamma\right)$, respectively, as follows

$$
\begin{aligned}
\mathcal{L}_{T}^{(1)} & =\frac{1}{4 m} \epsilon_{i j k} B^{j k} \dot{A}^{i}-\frac{1}{4 m} \epsilon_{i j k} A^{k} \dot{B}^{i j}-2 c_{2} \theta \dot{A}^{0}+\pi_{\theta} \dot{\theta}-2 c_{4} Q_{i} \dot{B}^{0 i}+P_{i} \dot{Q}^{i} \\
& +\phi_{\epsilon_{1}} \dot{\alpha}+\phi_{\vec{\epsilon}_{2}} \dot{\beta}^{i}+\phi_{\epsilon_{3}} \dot{\gamma}-\mathcal{H}_{T}^{(1)},
\end{aligned}
$$


where $\mathcal{H}_{T}^{(1)}=\left.\mathcal{H}^{(0)}\right|_{\phi_{\epsilon_{3}}=0}$ is the first-iterated Hamiltonian. We have now for the first-level symplectic variables $\xi^{(1) \alpha}$ and their conjugate momenta $a_{\alpha}^{(1)}$

$$
\begin{aligned}
\xi^{(1) \alpha} & =\left(A^{i}, B^{i j}, \theta, \pi_{\theta}, Q^{i}, P_{i}, A^{0}, B^{0 i}, \alpha, \beta^{i}, \gamma\right), \\
a_{\alpha}^{(1)} & =\left(\frac{1}{4 m} \epsilon_{i j k} B^{j k},-\frac{1}{2 m} \epsilon_{i j k} A^{k}, \pi_{\theta}, 0, P_{i}, \overrightarrow{0}^{T}, 0, \overrightarrow{0}^{T}, \phi_{\epsilon_{1}}, \phi_{\vec{\epsilon}_{2}}, \phi_{\epsilon_{3}}\right),
\end{aligned}
$$

and the first-iterated symplectic matrix now reads as

$$
f_{\alpha \beta}^{(1)}(x, y)=\left(\begin{array}{cc}
f_{\hat{\alpha} \hat{\beta}}^{(0)} & M_{\hat{\alpha} \sigma} \\
-M_{\sigma \hat{\alpha}}^{T} & O
\end{array}\right) \delta(x-y),
$$

where the submatrix $f_{\hat{\alpha} \hat{\beta}}^{(0)}$ refers to the $\xi_{\hat{\alpha}}=\left(A^{i}, B^{i j}, \theta, \pi_{\theta}, Q^{i}, P_{i}, A^{0}, B^{0 i}\right)$ sector, and $M_{\hat{\alpha} \sigma}$ is a $18 \times 5$ matrix defined as $M_{\hat{\alpha} \sigma}=\frac{\partial \phi_{\sigma}(y)}{\partial \xi_{\hat{\alpha}}(x)}$ :

$$
M_{\hat{\alpha} \sigma}(x, y)=\left(\begin{array}{ccc}
\overrightarrow{0} & -\frac{1}{m} \epsilon_{i j k} \partial_{y}^{k} & \overrightarrow{0} \\
\frac{1}{m} \epsilon_{i j k} \partial_{y}^{k} & O & \overrightarrow{0} \\
0 & \overrightarrow{0}^{T} & 0 \\
\frac{c_{2}}{2 c_{1}} & \overrightarrow{0}^{T} & 0 \\
\overrightarrow{0} & O & \overrightarrow{0} \\
\overrightarrow{0} & -\frac{c_{4}}{2 c_{3}} \delta_{i j} & \partial_{y}^{i} \\
1 & \overrightarrow{0}^{T} & 0 \\
\overrightarrow{0} & -\delta_{i j} & 2 c_{4} \partial_{i}^{y}
\end{array}\right) \delta(x-y)
$$

Then, this symplectic matrix, which contains no null rows and columns, is seemingly non-singular. Note that we observe the $Q^{0}$ term does not appear in the canonical sector of the Lagrangian $\mathcal{L}^{(1)}$ as well as in the Hamiltonian $\mathcal{H}_{T}^{(1)}$ due to the use of the constraint $\phi_{\epsilon_{3}}$. It is also important to eliminate the $Q^{0}$ field in the first-iterated level in the technical point of view. Keeping $Q^{0}$ field in the Lagrangian $\mathcal{L}^{(1)}$ makes trouble in the symplectic matrix because there is always zero row or column due to the absence of its conjugate momentum in $a^{(1)}(\xi)$. It prevents us from finding desired zero modes.

Now, the essential point of the symplectic Lagrangian embedding $[12,13$, $14,15]$ is this: in order to realize a gauge symmetry in this approach, the matrix (3.12) must have at least one "trivial" zero mode which does not generate a new constraint. In our case, the solution of

$$
\int \mathrm{d} y f_{\alpha \beta}^{(1)}(x, y) \nu_{\beta}^{(1)}(y)=0
$$


yields the following "trivial" zero modes

$$
\begin{aligned}
& \nu_{\alpha}^{(1) T}\left(\epsilon_{1}, x\right)=\left(\overrightarrow{0}^{T}, \overrightarrow{0}^{T}, 0,-c_{2} \epsilon_{1}, \overrightarrow{0}^{T}, \overrightarrow{0}^{T}, \epsilon_{1}, \overrightarrow{0}^{T}, 0, \overrightarrow{0}^{T}, 0\right), \\
& \nu_{\alpha}^{(1) T}\left(\vec{\epsilon}_{2}, x\right)=\left(\overrightarrow{0}^{T}, \overrightarrow{0}^{T}, 0,0, \overrightarrow{0}^{T}, 2 c_{4} \vec{\epsilon}_{2}, 0, \vec{\epsilon}_{2}, 0, \overrightarrow{0}^{T}, 0\right), \\
& \nu_{\alpha}^{(1) T}\left(\epsilon_{3}, x\right)=\left(\partial^{i} \epsilon_{3}, \overrightarrow{0}^{T},-\frac{1}{c_{2}} \epsilon_{3}, 0, \overrightarrow{0}^{T}, \overrightarrow{0}^{T}, 0, \overrightarrow{0}^{T}, \epsilon_{3}, \overrightarrow{0}^{T}, 0\right), \\
& \nu_{\alpha}^{(1) T}\left(\epsilon_{4}^{i}, x\right)=\left(\overrightarrow{0}^{T}, F_{24}^{T} \vec{\epsilon}_{4}, 0,0,-\frac{1}{2 c_{4}} \vec{\epsilon}_{4}, \overrightarrow{0}^{T}, 0, \overrightarrow{0}^{T}, 0, \vec{\epsilon}_{4}, 0\right), \\
& \nu_{\alpha}^{(1) T}\left(\epsilon_{5}, x\right)=\left(\overrightarrow{0}^{T}, \overrightarrow{0}^{T}, 0,0, \partial_{i} \epsilon_{5}, \overrightarrow{0}^{T}, 0, \overrightarrow{0}^{T}, 0, \overrightarrow{0}^{T}, \epsilon_{5}\right),
\end{aligned}
$$

where $F_{24}^{T}$ is defined in Eq. (2.20), while giving the relations for the free adjustable coefficients

$$
c_{2}^{2}=2 c_{1}, \quad c_{4}^{2}=-c_{3},
$$

which are obtained from the single-valuedness of the zero mode functions.

For consistency, we can confirm that these zero modes do not really generate any new constraint provided we apply them to the right-hand side of the equations of motion

$$
\int \mathrm{d} x \nu_{\alpha}^{(1)}(\sigma, x) \frac{\partial}{\partial \xi^{(1) \alpha}(x)} \int \mathrm{d} y \mathcal{H}_{T}^{(1)}(y)=0, \quad \sigma=\left(\epsilon_{1}, \vec{\epsilon}_{2}, \epsilon_{3}, \vec{\epsilon}_{4}, \epsilon_{5}\right),
$$

explicitly as

$$
\begin{aligned}
\int \mathrm{d} x \nu_{\alpha}^{(1) T}\left(\epsilon_{1}, x\right) \frac{\delta}{\delta \xi^{(1) \alpha}(x)} \int \mathrm{d} y \mathcal{H}^{(1)}(y) & =-\int \mathrm{d} x \epsilon_{1} \phi_{\epsilon_{1}}=0 \\
\int \mathrm{d} x \nu_{\alpha}^{(1) T}\left(\vec{\epsilon}_{2}, x\right) \frac{\delta}{\delta \xi^{(1) \alpha}(x)} \int \mathrm{d} y \mathcal{H}^{(1)}(y) & =\int \mathrm{d} x \vec{\epsilon}_{2} \phi_{\vec{\epsilon}_{2}}=0 \\
\int \mathrm{d} x \nu_{\alpha}^{(1) T}\left(\epsilon_{3}, x\right) \frac{\delta}{\delta \xi^{(1) \alpha}(x)} \int \mathrm{d} y \mathcal{H}^{(1)}(y) & =\int \mathrm{d} x \epsilon_{3}\left(1-\frac{c_{2}^{2}}{2 c_{1}}\right) \partial_{i} A^{i}=0 \\
\int \mathrm{d} x \nu_{\alpha}^{(1) T}\left(\epsilon_{4}, x\right) \frac{\delta}{\delta \xi^{(1) \alpha}(x)} \int \mathrm{d} y \mathcal{H}^{(1)}(y) & =-\int \mathrm{d} x \epsilon_{4}^{i}\left(1+\frac{c_{4}^{2}}{c_{3}}\right) \partial^{j} B_{i j}=0 \\
\int \mathrm{d} x \nu_{\alpha}^{(1) T}\left(\epsilon_{5}, x\right) \frac{\delta}{\delta \xi^{(1) \alpha}(x)} \int \mathrm{d} y \mathcal{H}^{(1)}(y) & =0
\end{aligned}
$$

where the third and fourth equations are reconfirmed by using the relations in Eq. (3.15). Since the last equation concerning the zero mode $\epsilon_{5}$ identically vanishes, this false zero mode plays no role at all, reflecting that the symplectic scheme deals only with true constraints. 
Therefore, before proceeding further to the symplectic embedding algorithm, with these determination of the coefficients we should go back to the first-iterated level (3.10) because the constraints are now not all independent, i.e., we can identify the last two constraints in Eq. (3.9) as

$$
\partial^{i} \phi_{\epsilon_{2}^{i}}-\frac{1}{2 c_{4}} \phi_{\epsilon_{3}}=0
$$

which means a gauge invariant Lagrangian for what we are seeking is reducible. In this case, in order for resolving the reducible constraint, we have to modify the auxiliary field as $\beta^{i} \rightarrow \beta^{i}-2 c_{4} \partial^{i} \gamma$. Then, a pair of the symplectic variable and the momentum, $\left(\gamma, \phi_{\epsilon_{3}}\right)$, are absorbed into the modified $\beta^{i}$ variables. This also modifies the symplectic matrix (3.12) to a new one which does not have the last row and column like the one in (3.7). Finding solutions having new zero modes is exactly equivalent in the corresponding "trivial" zero modes (3.16) to set the $\epsilon_{5}$-parameter zero. This clearly explains why the last zero mode in Eq. (3.14) does not generate any new constraints but vanishes identically.

On the other hand, we could also interpret these results in view of linear dependence of the "trivial" zero modes as follows: In the zero mode solutions (3.14) we have explicitly inserted the gauge parameters $\epsilon_{\sigma}$ keeping the form of gauge transformations (3.21) in mind. Instead, since we could normalize them by introducing the delta functional ${ }^{7}$ in the symplectic embedding scheme [14], we then have the last two zero modes in Eq. (3.14) explicitly as

$$
\begin{aligned}
& \nu_{\hat{\alpha}, y}^{(1) T}\left(\vec{\epsilon}_{4}, x\right)=\left(\overrightarrow{0}^{T}, F_{24}^{T}, 0,0,-\frac{1}{2 c_{4}} \vec{e}, \overrightarrow{0}^{T}, 0, \overrightarrow{0}^{T}\right) \delta(x-y), \\
& \nu_{\hat{\alpha}, y}^{(1) T}\left(\epsilon_{5}, x\right)=\left(\overrightarrow{0}^{T}, \overrightarrow{0}^{T}, 0,0, \nabla \epsilon_{5}, \overrightarrow{0}^{T}, 0, \overrightarrow{0}^{T}\right) \delta(x-y),
\end{aligned}
$$

where $\hat{\alpha}$ denote the component fields as $\xi_{\hat{\alpha}}=\left(A^{i}, B^{i j}, \theta, \pi_{\theta}, Q^{i}, P_{i}, A^{0}, B^{0 i}\right)$ and $\vec{e}$ is a unit 3 -dimensional vector. Then, we easily see that the zero mode concerning with the gauge parameter $\epsilon_{5}$ is related to $\nu_{\hat{\alpha}, y}^{(1) T}\left(\vec{\epsilon}_{4}, x\right)$ as

$$
\nu_{\hat{\alpha}, y}^{(1) T}\left(\epsilon_{5}, x\right)=-2 c_{4} \nabla \nu_{\hat{\alpha}, y}^{(1) T}\left(\vec{\epsilon}_{4}, x\right)
$$

${ }^{7}$ We can also label the zero modes as follows: $\nu_{\alpha, y}^{(l)}(\sigma, x),(\sigma=1, \ldots, N)$, where "l" refers to the "level", $\alpha, y$ stand for the component, while $\sigma, x$ label the $N$-fold infinity of zero modes in $\mathcal{R}^{3}$. See more details in Ref. [14]. 
showing that it is not linearly independent, i.e., reducible. In this point of view, we would call it, $\nu_{\hat{\alpha}, y}^{(1) T}\left(\epsilon_{5}, x\right)$, as a 'first-stage reducible' trivial zero mode. This extends previously known results $[12,13,14,15]$ in which the existence of "trivial" zero modes, or equivalently no new constraints are generated in the symplectic framework, implies gauge symmetry in system to include the first-stage reducible case.

As a result, we arrive at the final result on the symplectic embedding of the massive vector-tensor theory with topological coupling. The desired gauge invariant Lagrangian is now explicitly written as

$$
\begin{aligned}
\mathcal{L}_{T} & =\mathcal{L}_{O}+\mathcal{L}_{W Z} \\
& =-\frac{1}{4}\left(B_{\mu \nu}-2 c_{4} Q_{\mu \nu}\right)^{2}+\frac{1}{2}\left(A_{\mu}+c_{2} \partial_{\mu} \theta\right)^{2}+\frac{1}{2 m} \epsilon_{\mu \nu \rho \sigma} B^{\mu \nu} \partial^{\rho} A^{\sigma} .
\end{aligned}
$$

This ends the Lagrangian embedding in the symplectic setup.

Now, in order to discuss gauge transformation, we consider the symplectic matrix (3.12). As has been shown in Ref. [9, 12], the "trivial" zero modes generate gauge transformations on the symplectic variable $\xi_{\hat{\alpha}}^{(1)}$ in the sense

$$
\delta \xi_{\alpha}(x)=\Sigma_{\tilde{\sigma}} \nu_{\alpha}^{(1) T}(\tilde{\sigma}, x), \quad \tilde{\sigma}=\left(\epsilon_{1}, \vec{\epsilon}_{2}, \epsilon_{3}, \vec{\epsilon}_{4}\right) .
$$

We thus obtain the gauge transformations of the symplectic variables from Eq. (3.14) as follows

$$
\begin{aligned}
\delta A^{0} & =\epsilon_{1}, \quad \delta A^{i}=\partial^{i} \epsilon_{3}, \quad \delta \theta=-\frac{1}{c_{2}} \epsilon_{3}, \\
\delta B^{0 i} & =\epsilon_{2}^{i}, \quad \delta B^{i j}=-\left(\partial^{i} \epsilon_{4}^{j}-\partial^{j} \epsilon_{4}^{i}\right), \quad \delta Q^{i}=-\frac{1}{2 c_{4}} \epsilon_{4}^{i},
\end{aligned}
$$

except the missing transformation $\delta Q^{0}$ which cannot be obtained at this stage due to the elimination of the $Q^{0}$ component in the first-iterated level of the procedure. This is very similar with the BFT Hamiltonian embedding for the constrained reducible system in which we should introduce the $Q^{0}$ field in the path integral measure in order to keep manifest covariance in the Lagrangian $^{8}$.

\footnotetext{
${ }^{8}$ We will discuss this explicitly in the next section.
} 
To find out explicitly the gauge transformation for the $Q^{0}$ field as well as any existence of possible restrictions to the gauge parameters, let us consider the total variation of the Lagrangian (3.20), which transforms as

$$
\begin{aligned}
\delta \mathcal{L}_{T} & =\left(A_{0}+c_{2} \partial_{0} \theta\right)\left(\epsilon_{1}-\partial^{0} \epsilon_{3}\right) \\
& +\left(\frac{1}{m} \epsilon_{i j k} \partial^{j} A^{k}-B_{0 i}+2 c_{4} Q_{0 i}\right)\left(\epsilon_{2}^{i}+\partial^{0} \epsilon_{4}^{i}+2 c_{4} \partial^{i} \delta Q^{0}\right) .
\end{aligned}
$$

Therefore, if we identify the gauge parameters as

$$
\epsilon_{1}=\partial^{0} \epsilon_{3}, \quad \epsilon_{2}^{i}=-\left(\partial^{0} \epsilon_{4}^{i}-\partial^{i} \epsilon_{4}^{0}\right)
$$

along with a new parameter $\epsilon_{4}^{0}$ defined as

$$
\delta Q^{0}=-\frac{1}{2 c_{4}} \epsilon_{4}^{0},
$$

we can make the total Lagrangian $\mathcal{L}_{T}$ gauge invariant. As a result, this Lagrangian is invariant under the final gauge transformations as

$$
\begin{aligned}
& \delta A^{\mu}=\partial^{\mu} \epsilon_{3}, \quad \delta \theta=-\frac{1}{c_{2}} \epsilon_{3}, \\
& \delta B^{\mu \nu}=-\left(\partial^{\mu} \epsilon_{4}^{\nu}-\partial^{\nu} \epsilon_{4}^{\mu}\right), \quad \delta Q^{\mu}=-\frac{1}{2 c_{4}} \epsilon_{4}^{\mu} .
\end{aligned}
$$

Therefore, by considering purely the symplectic Lagrangian embedding, we have found the gauge invariant Lagrangian as well as their full gauge transformations including $\delta Q^{0}$. Note that the coefficients $c_{2}, c_{4}$ can be either rescaled on the variables as $\left(\theta, Q^{\mu}\right) \rightarrow\left(c_{2} \theta, 2 c_{4} Q^{\mu}\right)$, or fixed as $c_{2}= \pm 1, c_{4}= \pm \frac{1}{2}$. Then, the resulting Lagrangian is reduced to the well-known gauge embedded form of the massive vector-tensor theory with topological coupling,

$$
\mathcal{L}_{T}=-\frac{1}{4}\left(B_{\mu \nu}-Q_{\mu \nu}\right)^{2}+\frac{1}{2}\left(A_{\mu}+\partial_{\mu} \theta\right)^{2}+\frac{1}{2 m} \epsilon_{\mu \nu \rho \sigma} B^{\mu \nu} \partial^{\rho} A^{\sigma},
$$

where we set the free adjustable coefficients as $c_{2}=1$ and $c_{4}=1 / 2$, and this Lagrangian is invariant under

$$
\begin{aligned}
& \delta A^{\mu}=\partial^{\mu} \epsilon, \quad \delta \theta=-\epsilon, \\
& \delta B^{\mu \nu}=\partial^{\mu} \epsilon^{\nu}-\partial^{\nu} \epsilon^{\mu}, \quad \delta Q^{\mu}=\epsilon^{\mu},
\end{aligned}
$$

where the gauge parameters are redefined as $\epsilon=\epsilon_{3}$ and $\epsilon^{\mu}=-\epsilon_{4}^{\mu}$. 


\section{Revisited BFT Embedding}

In this section, we will compare the symplectic Lagrangian embedding with the previous work [7] of the BFT Hamiltonian one, which makes secondclass constraint Hamiltonian system into corresponding first-class one in a systematic way.

First, let us start with the canonical momenta from the symmetrized form of the Lagrangian (2.1) as $\pi_{0}=0, \pi_{i}=\frac{1}{4 m} \epsilon_{i j k} B^{j k}, \pi_{0 i}=0$, and $\pi_{i j}=$ $-\frac{1}{2 m} \epsilon_{i j k} A^{k}$ in order to analyze the Hamiltonian structure of the model. Then, we have obtained the primary Hamiltonian in the Dirac's terminology as

$$
\begin{aligned}
\mathcal{H}_{p} & =\frac{1}{4} B_{\mu \nu} B^{\mu \nu}-\frac{1}{2} A_{\mu} A^{\mu}-\frac{1}{m} \epsilon^{i j k} B_{0 i} \partial_{j} A_{k}-\frac{1}{2 m} A^{0} \epsilon^{i j k} \partial_{i} B_{j k} \\
& +\lambda^{0} \pi_{0}+\lambda^{i} \Omega_{i}+\Sigma^{0 i} \pi_{0 i}+\Sigma^{i j} \Omega_{i j}
\end{aligned}
$$

where the ten primary constraints are given by

$$
\begin{aligned}
& \pi_{0} \approx 0, \quad \Omega_{i} \equiv \pi_{i}-\frac{1}{4 m} \epsilon_{i j k} B^{j k} \approx 0 \\
& \pi_{0 i} \approx 0, \quad \Omega_{i j} \equiv \pi_{i j}+\frac{1}{2 m} \epsilon_{i j k} A^{k} \approx 0
\end{aligned}
$$

along with their associated Lagrange multipliers $\lambda^{0}, \lambda^{i}, \Sigma^{0 i}$, and $\Sigma^{i j}$.

There are four additional secondary constraints which are obtained from the time stability conditions of the primary constraints $\pi_{0}$ and $\pi_{0 i}$ as

$$
\begin{aligned}
\Lambda & \equiv A_{0}+\frac{1}{2 m} \epsilon_{i j k} \partial^{i} B^{j k} \approx 0, \\
\Lambda_{i} & \equiv B_{0 i}-\frac{1}{m} \epsilon_{i j k} \partial^{j} A^{k} \approx 0 .
\end{aligned}
$$

As expected, the Lagrange multipliers $\Sigma^{i j}, \lambda^{i}$ corresponding to the constraints $\Omega_{i}, \Omega_{i j}$ are fixed under the time stability condition, and the other ones $\lambda^{0}, \Sigma^{0 i}$ are determined by the consistency requirement of the secondary constraints $\Lambda, \Lambda_{i}$, respectively.

Then, the full set of these constraints makes the constraint algebra secondclass as

$$
\begin{aligned}
\left\{\pi_{0}, \Lambda\right\} & =-\delta(x-y), \\
\left\{\pi_{0 i}, \Lambda_{j}\right\} & =-\delta_{i j} \delta(x-y), \\
\left\{\Omega_{i}, \Omega_{j k}\right\} & =-\frac{1}{m} \epsilon_{i j k} \delta(x-y),
\end{aligned}
$$


where we have redefined the secondary constraints as $\Lambda+\partial^{i} \Omega_{i} \rightarrow \Lambda=\partial^{i} \pi_{i}+$ $A^{0}+\frac{1}{4 m} \epsilon^{i j k} \partial_{i} B_{j k} \approx 0$, and $\Lambda_{i}+\partial^{j} \Omega_{i j} \rightarrow \Lambda_{i}=\partial^{j} \pi_{i j}+B_{0 i}-\frac{1}{2 m} \epsilon_{i j k} \partial^{j} A^{k} \approx 0$. These new definitions make the constraint algebra have no derivative terms. As a result, we have easily obtained the following Dirac Brackets

$$
\begin{aligned}
\left\{A^{0}(x), A^{i}(y)\right\}_{D} & =\partial_{x}^{i} \delta(x-y), \\
\left\{A^{i}(x), B^{j k}(y)\right\}_{D} & =-m \epsilon^{i j k} \delta(x-y), \\
\left\{B^{0 i}(x), B^{j k}(y)\right\}_{D} & =\left(\delta^{i j} \partial_{x}^{k}-\delta^{i k} \partial_{x}^{j}\right) \delta(x-y), \\
\left\{A^{i}(x), \pi_{j}(y)\right\}_{D} & =\frac{1}{2} \delta_{j}^{i} \delta(x-y), \\
\left\{A^{0}(x), \pi_{i j}(y)\right\}_{D} & =-\frac{1}{2 m} \epsilon_{i j k} \partial_{x}^{k} \delta(x-y), \\
\left\{\pi_{i}(x), B_{0 j}(y)\right\}_{D} & =\frac{1}{2 m} \epsilon_{i j k} \partial_{x}^{k} \delta(x-y), \\
\left\{\pi_{i}(x), \pi_{j k}(y)\right\}_{D} & =\frac{1}{4 m} \epsilon_{i j k} \delta(x-y), \\
\left\{B^{i j}(x), \pi_{k l}(y)\right\}_{D} & =\frac{1}{2}\left(\delta_{k}^{i} \delta_{l}^{j}-\delta_{k}^{j} \delta_{l}^{i}\right) \delta(x-y)
\end{aligned}
$$

for the massive vector-tensor theory with topological coupling.

Now, let us briefly recapitulate the BFT Hamiltonian embedding for this theory. In order for that purpose, we have introduced auxiliary fields having involutive relations in which not only modified new constraints in the enlarged space are strongly vanishing with each other, but also they have the vanishing Poisson brackets, not the Dirac brackets, with physical quantities such as Hamiltonian and fields themselves. Through this BFT prescription, after introducing auxiliary fields paired as $\left(\theta, \pi_{\theta}\right),\left(Q^{i}, P_{i}\right)$, and $\left(\Phi^{i}, \Phi^{j k}\right)$, we have obtained the strongly involutive primary

$$
\begin{aligned}
& \tilde{\pi}_{0}=\pi_{0}+\theta, \quad \tilde{\pi}_{0 i}=\pi_{0 i}+Q_{i}, \\
& \widetilde{\Omega}_{i}=\Omega_{i}+\Phi_{i}, \quad \widetilde{\Omega}_{i j}=\Omega_{i j}+\frac{1}{m} \Phi_{i j},
\end{aligned}
$$

and secondary constraints

$$
\begin{aligned}
\widetilde{\Lambda} & =\Lambda+\pi_{\theta}=A_{0}+\frac{1}{2 m} \epsilon_{i j k} \partial^{i} B^{j k}+\pi_{\theta}, \\
\widetilde{\Lambda}_{i} & =\Lambda_{i}+P_{i}=B_{0 i}-\frac{1}{m} \epsilon_{i j k} \partial^{j} A^{k}+P_{i} .
\end{aligned}
$$


Moreover, the strongly involutive physical fields are also obtained as

$$
\begin{aligned}
& \widetilde{A}^{0}=A^{0}+\pi_{\theta}, \widetilde{A}^{i}=A^{i}+\partial^{i} \theta-\frac{1}{2} \epsilon^{i j k} \Phi_{j k}, \\
& \widetilde{\pi}_{0}=\pi_{0}+\theta, \quad \widetilde{\pi}_{i}=\pi_{i}-\frac{1}{2 m} \epsilon_{i j k} \partial^{j} Q^{k}+\frac{1}{2} \Phi_{i}, \\
& \widetilde{B}^{0 i}=B^{0 i}+P^{i}, \quad \widetilde{B}^{i j}=B^{i j}-\left(\partial^{i} Q^{j}-\partial^{j} Q^{i}\right)+m \epsilon^{i j k} \Phi_{k}, \\
& \widetilde{\pi}_{0 i}=\pi_{0 i}+Q_{i}, \widetilde{\pi}_{i j}=\pi_{i j}-\frac{1}{2 m} \epsilon_{i j k} \partial^{k} \theta+\frac{1}{2 m} \Phi_{i j} .
\end{aligned}
$$

Note that all these physical fields are terminated in the first order of the auxiliary fields, and the Poisson brackets of these fields in the extended phase space are exactly same as the Dirac brackets (4.5) in the original phase space.

On the other hand, by using the strongly involutive physical fields (4.8), we have also obtained the extended canonical Hamiltonian as

$$
\begin{aligned}
\widetilde{\mathcal{H}}_{c} & =\frac{1}{4}\left(B_{i j}-Q_{i j}\right)^{2}+\frac{1}{2} m \epsilon_{i j k}\left(B^{i j}-Q^{i j}\right) \Phi^{k}-\frac{1}{2} m^{2} \Phi_{i} \Phi^{i} \\
& -\frac{1}{2}\left(A_{i}+\partial_{i} \theta\right)^{2}+\frac{1}{2} \epsilon_{i j k}\left(A^{i}+\partial^{i} \theta\right) \Phi^{j k}+\frac{1}{4} \Phi_{i j} \Phi^{i j} \\
& +\frac{1}{2}\left(B_{0 i}+P_{i}\right)^{2}-\frac{1}{m} \epsilon_{i j k}\left(B^{0 i}+P^{i}\right) \partial^{j} A^{k}-\frac{1}{m}\left(B_{0 i}+P_{i}\right) \partial_{j} \Phi^{i j} \\
& -\frac{1}{2}\left(A_{0}+\pi_{\theta}\right)^{2}-\frac{1}{2 m}\left(A_{0}+\pi_{\theta}\right) \epsilon_{i j k} \partial^{i} B^{j k}+\left(A_{0}+\pi_{\theta}\right) \partial_{i} \Phi^{i}, \\
& +\pi_{\theta} \widetilde{\Lambda}+P_{i} \widetilde{\Lambda}_{i},
\end{aligned}
$$

where the last two terms are added to generate the Gauss' constraints corresponding to the constraints, $\widetilde{\pi}_{0}, \widetilde{\pi}_{0 i}$, consistently. Then, the generating functional for the extended first-class systems is given by

$$
\begin{aligned}
\mathcal{Z} & =\int \mathcal{D} A^{\mu} \mathcal{D} \pi_{\mu} \mathcal{D} B^{\mu \nu} \mathcal{D} \pi_{\mu \nu} \mathcal{D} \theta \mathcal{D} \pi_{\theta} \mathcal{D} Q^{i} \mathcal{D} P_{i} \mathcal{D} \Phi^{i} \mathcal{D} \Phi^{i j} \\
& \times \delta\left(\widetilde{\varphi}_{\alpha}\right) \delta\left(\Gamma_{\beta}\right) \operatorname{det}\left|\left\{\widetilde{\varphi}_{\alpha}, \Gamma_{\beta}\right\}\right| e^{i S},
\end{aligned}
$$

where

$$
S=\int d^{4} x\left[\pi_{\mu} \dot{A}^{\mu}+\pi_{0 i} \dot{B}^{0 i}+\frac{1}{2} \pi_{i j} \dot{B}^{i j}+\pi_{\theta} \dot{\theta}+P_{i} \dot{Q}^{i}+\frac{1}{2} \epsilon_{i j k} \Phi^{i} \dot{\Phi}^{j k}-\widetilde{\mathcal{H}}_{c}\right],
$$


and $\Gamma_{\alpha}$ are appropriate gauge fixing functions which have nonvanishing Poisson brackets with the modified first-class constraints $\tilde{\varphi}_{\alpha}=\left(\widetilde{\pi}_{0}, \widetilde{\pi}_{0 i}, \widetilde{\Lambda}, \widetilde{\Lambda}_{i}, \widetilde{\Omega}_{i}, \widetilde{\Omega}_{i j}\right)$.

Now, by performing the momenta integrations in the generating functional as usual [14], we have finally obtained the gauge invariant Lagrangian corresponding to $\widetilde{\mathcal{H}}_{c}$ as

$$
\mathcal{Z}=\int \mathcal{D} A^{\mu} \mathcal{D} B^{\mu \nu} \mathcal{D} \theta \mathcal{D} Q^{\mu} \mathcal{D} \Phi^{i} \mathcal{D} \Phi^{i j} \delta\left(Q^{0}\right) \delta\left(\Gamma_{\beta}\right) \operatorname{det}\left|\left\{\widetilde{\varphi}_{\alpha}, \Gamma_{\beta}\right\}\right| e^{i S_{T}}
$$

where

$$
\begin{aligned}
S_{T} & =\int d^{4} x\left(\mathcal{L}_{G E}+\mathcal{L}_{N W Z}\right) \\
\mathcal{L}_{G E} & =-\frac{1}{4}\left(B_{\mu \nu}-Q_{\mu \nu}\right)^{2}+\frac{1}{2}\left(A_{\mu}+\partial_{\mu} \theta\right)^{2}+\frac{1}{2 m} \epsilon_{\mu \nu \rho \sigma} B^{\mu \nu} \partial^{\rho} A^{\sigma} \\
\mathcal{L}_{N W Z} & =\frac{1}{2} \epsilon_{i j k} \Phi^{i} \dot{\Phi}^{j k}+\left[F_{i 0}-\frac{m}{2} \epsilon_{i j k}\left(B^{j k}-Q^{j k}\right)+\frac{m^{2}}{2} \Phi_{i}\right] \Phi^{i} \\
& -\left[\frac{1}{2} \epsilon_{i j k}\left(A^{i}+\partial^{i} \theta\right)+\frac{1}{m} \partial_{k} B_{0 j}+\frac{1}{2 m} \partial_{0} B_{j k}+\frac{1}{4} \Phi_{i j}\right] \Phi^{j k}
\end{aligned}
$$

where $\mathcal{L}_{G E}$ is the gauge embedded Lagrangian and $\mathcal{L}_{N W Z}$ is a new type of the WZ Lagrangian. Note that we have introduced the delta functional of a variable $Q^{0}$ in the measure which serves the final gauge embedded Lagrangian $\mathcal{L}_{G E}$ manifestly covariant.

On the other hand, the infinitesimal gauge transformations for the fields are given as

$$
\begin{aligned}
\delta A^{\mu} & =-\partial^{\mu} \epsilon_{A}+\delta_{j}^{\mu} \epsilon_{A}^{j}, & & \delta B^{\mu \nu}=\partial^{\mu} \epsilon_{B}^{\nu}-\partial^{\nu} \epsilon_{B}^{\mu}+\left(\epsilon_{B}^{k l}-\epsilon_{B}^{l k}\right) \delta_{k}^{\mu} \delta_{l}^{\nu}, \\
\delta \theta & =\epsilon_{A}, & & \delta Q^{\mu}=\epsilon_{B}^{\mu}, \\
\delta \Phi^{i} & =\frac{1}{m} \epsilon^{i j k} \epsilon_{j k}^{B}, & & \delta \Phi^{i j}=-\epsilon^{i j k} \epsilon_{k}^{A}
\end{aligned}
$$

from the generator of the gauge transformation ${ }^{9}$, the first-class constraints (4.6). The transformations related to the gauge symmetry are exactly same as the ones (3.26) obtained from the symplectic Lagrangian scheme. On the other hand, the other parameters $\epsilon_{A}^{i}, \epsilon_{B}^{i j}$ in Eq. (4.15) are not related with the gauge symmetry. These parameters are associated with the symplectic structure of the topological coupling, which are absent in the symplectic

\footnotetext{
${ }^{9}$ See Ref.[7] for details.
} 
Lagrangian scheme. Since in the latter scheme the topological coupling term is already of first ordered, we have no need to introduce the auxiliary fields (momenta) which have become the gauge generators in the former scheme. As a result, the new type of the WZ Lagrangian, which may lack manifest covariance, is indeed invariant under the extended transformations including these parameters. Moreover, by fixing unitary gauge conditions such as $\Phi^{i}=$ $\Phi^{i j}=0$, the new type of the WZ Lagrangian identically vanishes, while the strongly involutive constraints $\tilde{\Lambda}, \tilde{\Lambda}^{i}$ in Eq. (4.6) become exactly same with the true ones $\phi_{\epsilon_{1}}, \phi_{\vec{\epsilon}_{2}}$ in Eq. (3.9) generated from the symplectic embedding procedure, respectively.

It is also important to note that in the generating functional (4.12) there exists the delta functional of the variable $Q^{0}$ which transforms as $\delta Q^{0}=\epsilon_{B}^{0}$. We have introduced this new field to make the final Lagrangian manifestly covariant. Even without this $Q^{0}$ field, we can show that the resulting Lagrangian successfully reproduces all the BFT embedded constraint structure. However, in that case, it will lose manifest covariance. Indeed, we have introduced the new variable $Q^{0}$ in order for keeping the manifest covariance, while giving up the irreducible property between the constraints [7].

\section{Conclusion}

In this paper we have quantized a massive vector-tensor theory with topological coupling which is a first order master action of the Proca and the Kalb-Ramond models. We have adopted the symplectic scheme since it provides relatively simple way of getting the Dirac brackets rather than that of Dirac for the Lagrangian having seemingly complicated antisymmetric tensor fields. In particular, we have shown that our model has the only four constraints in the symplectic scheme in contrast to the Dirac (or, BFT) formalism having the fourteen constraints. Moreover, we have demonstrated how the BFT embedding scheme of second-class system into first-class one can be realized in the framework of the symplectic approach to constrained system. Rather than proceeding iteratively as in the BFT embedding approach, we have greatly simplified the calculation by making use of manifest Lorentz invariance in our Ansatz for the WZ term.

Furthermore, we have explicitly shown that the reducibility between the constraints in the resulting gauge invariant Lagrangian comes from the ab- 
sence of the $Q^{0}$ term, which naturally arises in analyzing the model's symplectic structure. Requiring the gauge invariance of the total Lagrangian, we have successfully recovered it and thus have obtained the full gauge transformations consistently, while showing that all the zero modes solutions are not linearly independent, i.e., there exists the first-stage reducible trivial zero mode.

Similar to the symplectic Lagrangian embedding, we have shown in the BFT Hamiltonian embedding that the reducibility between the constraints is also related to the $Q^{0}$ component, and have successfully reconstructed the Lorentz covariant gauge embedded Lagrangian by using the delta functional of the $Q^{0}$ field in the path integral measure. However, in this scheme which carries out the rigorous converting procedure from the second-class constraints to the effective first-class ones, due to the appearance of the constraints $\Omega_{i}, \Omega_{i j}$ in Eq. (4.6) originated from the symplectic structure of the theory, we can not keep the Lorentz covariance as seen in the new type of the WZ Lagrangian. Nevertheless, we have shown that the total action including the NWZ action is invariant under the extended gauge transformations. Compared with this, since in the symplectic Lagrangian embedding we do not need to classify the constraints as the second-, or the first-, we could freely require the Lorentz covariance without any inconsistency. In short, gauge embedded extension in the symplectic scheme is only related with the Lorentz covariant action while the usual symplectic scheme concerns only with true constraints.

Finally, the method followed by this work is worthwhile in itself because there is no known systematic method yet except the simplest one-form cases $[12,13,14]$. In this respect, we have newly generalized the symplectic Lagrangian embedding procedure to include highly non-trivial tensor fields which exhibit, for example, the existence of the first-stage reducible trivial zero mode among the others related to the reducibility of theory.

The work of YWK was supported by the Korea Research Foundation, Grant No. KRF-2002-075-C00007. The work of CYL was supported by KOSEF, Grant No. R01-2000-000-00022-0. 


\section{References}

[1] P. A. M. Dirac, Lectures on quantum mechanics (Belfer graduate School, Yeshiba University Press, New York, 1964).

[2] C. Becci, A. Rouet, and R. Stora, Ann. Phys. (N.Y.) 98, 287 (1976); I. V. Tyutin, Lebedev Preprint 39 (1975); T. Kugo and I. Ojima, Prog. Theor. Phys. Suppl. 66, 1 (1979).

[3] E. S. Fradkin and G. A. Vilkovisky, Phys. Lett. 55B, 224 (1975); I. A. Batalin and G. A. Vilkovisky, Phys. Lett. 69B, 309 (1977); M. Henneaux, Phys. Rept. 126, 1 (1985); T. Fujiwara, Y. Igarashi, and J. Kubo, Nucl. Phys. B341, 695 (1990); Y.-W. Kim, S.-K. Kim, W.T. Kim, Y.-J. Park, K.Y. Kim, and Y. Kim, Phys. Rev. D46, 4574 (1992).

[4] I. A. Batalin and E. S. Fradkin, Nucl. Phys. B279, 514 (1987); I. A. Batalin and I. V. Tyutin, Int. J. Mod. Phys. A6, 3255 (1991).

[5] R. Banerjee, Phys. Rev. D48, R5467 (1993); W. T. Kim and Y.-J. Park, Phys. Lett. B336, 376 (1994).

[6] Y.-W. Kim, Y.-J. Park, K. Y. Kim, and Y. Kim, Phys. Rev. D51, 2943 (1995); J.-H. Cha, Y.-W. Kim, Y.-J. Park, Y. Kim, S.-K. Kim, and W. T. Kim, Z. Phys. C69, 175 (1995); R. Amorim and J. Barcelos-Neto, Phys. Rev. D53, 7129 (1996); Y.-W. Kim and K. D. Rothe, Nucl. Phys. B510, 511 (1998); S. T. Hong and Y.-J. Park, Phys. Rept. 358, 143 (2002); S. T. Hong and S. H. Lee, Eur. Phys. J. C25, 131 (2002).

[7] Y.-W. Kim, S.-K. Kim, and Y.-J. Park, Mod. Phys. Lett. A18, 2287 (2003).

[8] L. Faddeev and R. Jackiw, Phys. Rev. Lett. 60, 1692 (1988).

[9] J. Barcelos-Neto and C. Wotzasek, Mod. Phys. Lett. A7, 1172 (1992); H. Montani, Int. J. Mod. Phys. A8, 3419 (1993); Y.-W. Kim, Y.-J. Park, K. Y. Kim, Y. D. Kim, and C.-H. Kim, J. Korean Phys. Soc. 26, 243 (1993).

[10] J. Barcelos-Neto and E. S. Cheb-Terrab, Z. Phys. C54, 133 (1992); J. Lukierski, P. C. Stichel, and W. J. Zakrzewski, Ann. Phys. 260, 224 
(1997); C. Y. Lee and D. W. Lee, J. Phys. A31, 7809 (1998); C. Duval and P. A. Horvathy, J. Phys. A34, 10097 (2001); H. J. Rothe and K. D. Rothe, J. Phys. A36, 1671 (2003).

[11] N. R. F. Braga and C. F. L. Godinho, Phys. Rev. D65, 085030 (2002); Z.-W. Long and J. Jing, Phys. Lett. B560, 128 (2003); J. Jing, Z.-W. Long, and L.-J. Tian, Eur. Phys. J. C29, 447 (2003).

[12] S.-K. Kim, Y.-W. Kim, Y.-J. Park, K. Y. Kim, Y. Kim, and W. T. Kim, J. Korean Phys. Soc. 28, 128 (1995).

[13] R. Banerjee and J. Barcelos-Neto, Ann. Phys. 265, 134 (1998); S.-T. Hong, Y.-W. Kim, Y.-J. Park, and K. D. Rothe, J. Phys. A35, 7461 (2002); J. A. Neto, C. Neves, and W. Oliveira, Int. J. Mod. Phys. A18, 1883 (2003).

[14] S.-T. Hong, Y.-W. Kim, Y.-J. Park, and K. D. Rothe, Mod. Phys. Lett. A17, 435 (2002).

[15] J. A. Neto, A. C. R. Mendes, C. Neves, W. Oliveira, and D. C. Rodrigues, hep-th/0109089; C. Neves, W. Oliveira, D. C. Rodrigues, and C. Wotzasek, hep-th/0311209.

[16] M. Kalb and P. Ramond, Phys. Rev. D9, 2273 (1974); A. Aurilia and Y. Takahashi, Prog. Theor. Phys. 66, 693 (1981).

[17] S. Deser, R. Jackiw and S. Templeton, Ann. Phys. (N.Y.) 140, 372 (1982).

[18] E. Cremmer and J. Scherk, Nucl. Phys. B72, 117 (1974); T. J. Allen, M. J. Bowick, and A. Lahiri, Mod. Phys. Lett. A6, 559 (1991); J. BarcelosNeto and S. Rabello, Z. Phys. C74, 715 (1997); D. S. Hwang and C.-Y. Lee, J. Math. Phys. 38, 30 (1997).

[19] S.-J. Rey, Phys. Rev. D40, 3396 (1989); E. Poppitz, Nucl. Phys. B542, 31 (1999); D. M. Ghilencea, L. E. Ibàñez, N. Irges, and F. Quevedo, JHEP 08, 016 (2002).

[20] A. Lahiri, Mod. Phys. Lett. A8, 2403 (1993); H. Sawayanagi, Mod. Phys. Lett. A10, 813 (1995); E. Harikumar and M. Sivakumar, Phys. 
Rev. D57, 3794 (1998); S. Deguchi, T. Mukai, and T. Nakajima, Phys. Rev. D59, 065003 (1999).

[21] N. Banerjee and R. Banerjee, Mod. Phys. Lett. A11, 1919 (1996).

[22] M. B. Cantcheff, Eur. Phys. J. C6, 1 (2002).

[23] S. Deser and R. Jackiw, Phys. Lett. 139B, 371 (1984); R. Gianvittorio, A. Restuccia, and J. Stephany, Mod. Phys. Lett. A6, 2121 (1991); R. Banerjee, H. J. Rothe, and K. D. Rothe, Phys. Rev. D55, 6339 (1997).

[24] E. Harikumar and M. Sivakumar, Nucl. Phys. B565, 385 (2000); Int. J. Mod. Phys. A17, 405 (2002).

[25] J. Wess and B. Zumino, Phys. Lett. B37, 95 (1971); L. D. Faddeev and S. L. Shatashivili, Phys. Lett. B167, 225 (1986).

[26] J. Barcelos-Neto and A. Cabo, Z. Phys. C74, 731 (1997). 